\title{
Transswitching as a means of studying within-subjects conditioning effects
}

\author{
H. D. KIMMEL and K. A. GARDNER \\ University of South Florida, Tampa, Florida 33620
}

\begin{abstract}
A within-subjects experiment is described in which the transswitching paradigm was used to evaluate the effect of CS-US pairing on conditioned and unconditioned responding. Three different tonic auditory stimuli served as contexts within which the CS was administered alone, the US was administered alone, or the CS and US were paired. The magnitudes of the CR and UR were both influenced by pairing (CR higher and UR lower in paired context). Tonic response differences were not significant. The results demonstrated the utility of the transswitching method for within-subjects conditioning experiments.
\end{abstract}

The transswitching conditioning paradigm (Asratyan, 1965; Kimmel \& Ray, 1978) has been employed primarily in the study of tonic stimulus control of tonic and phasic conditioned responses. The method has proved to be useful in elucidating the manner in which ongoing (tonic) environmental stimuli acquire a significant role in the differentiation of specific learned reactions to the same phasic conditioned stimulus in different experimental situations.

Because the tonic stimuli used in transswitching define separable tonic environmental contexts (Kimmel, 1979) within which the same phasic stimulus may be associated with different responses, the transswitching paradigm also provides a methodological tool for studying simple conditioning phenomena within a single group of experimental subjects. The subjects "serve as their own controls" in the sense that experimental and control treatments may be administered to them within the separate contexts provided by the tonic stimuli.

In the present study, for example, three different auditory tonic contexts were differentiated by the fact that a visual conditioned stimulus was sporadically administered alone in one of the auditory contexts, an electric shock unconditioned stimulus was sporadically administered alone in the second auditory context, and the light and shock were paired in the third auditory context. Differences between responses to the visual conditioned stimulus could be observed between the two auditory contexts containing visual stimuli, whereas differences between responses to the shock could be observed between the two auditory contexts containing shocks. In addition to these phasic response comparisons, which reflect indirect influences of the tonic auditory contexts, the procedure also provided an opportunity to observe tonic differences directly associated with the tonic auditory contexts.

It was expected that the visual conditioned stimulus would elicit larger conditioned skin conductance responses (SCRs) when it was presented in the auditory context in which it was paired with the shock than when it was presented in the context in which it was presented unpaired, although comparisons involving the same conditioned stimulus in different contexts obviously may be attenuated by generalized excitatory tendencies from the paired to the unpaired context, as well as by generalized inhibitory tendencies from the unpaired to the paired context. In order to provide enough training for conditioned differences to be seen, two separate conditioning sessions, 2 days apart, were run. It was further expected that the unconditioned SCR to the shock would be larger in magnitude in the context in which the shock was unpaired with the conditioned stimulus than in the context in which it was administered paired. Although we have previously found differences in phasic conditioned SCR magnitude between paired and unpaired contexts (Kimmel \& Ray, 1978), diminution of the unconditioned SCR (Kimmel, 1966) has not previously been studied using the transswitching paradigm. Finally, it was expected that the tonic auditory stimuli would acquire the capacity to control differences in tonic conditioned changes, depending upon the types of events that had been administered in their presence. Specifically, it was expected that the most aversive context would be that in which unsignaled shock occurred (shock alone), the next most aversive context would be that in which signaled shock occurred (light + shock), and the least aversive context would be that in which no shock occurred (light alone). To the extent that the tonic conditioned responses reflect nonspecific autonomic activation, it was assumed that the conditioned aversiveness of the auditory context would be manifested in increasing levels of autonomic activity, specifically, frequency of unelicited skin conductance responding.

\section{METHOD}

\section{Subjects}

Ten male introductory psychology students at the University of South Florida volunteered to serve as subjects to receive extra class points. All of the subjects were informed that the experi- 
ment involved occasional electric shocks and that its purpose was to measure physiological reactions to various stimuli. The subjects were told to relax, avoid unnecessary movements, and pay attention to the stimuli.

\section{Apparatus}

Palmar skin resistance was picked up from the palm and back of the subject's nonpreferred hand by $1.9-\mathrm{cm}$ zinc/zinc sulfate electrodes in Lucite cups filled with saline electrode paste. It was amplified by a Biophysical Instrument Company amplifier and recorded on a Texas Instruments Recti-Riter with a paper speed of $15.24 \mathrm{~cm} / \mathrm{min}$. Phasic response magnitudes were transformed to units of change in log conductance. The tonic auditory stimuli were $500-, 800-$, and $1,400-\mathrm{Hz}$ pure tones at approximately $40 \mathrm{~dB}$ (physical reference), produced by a General Radio Company oscillator-amplifier and delivered via wall-mounted speakers in front of and behind the subject in order to create an effect of completely surrounding sound. The duration of each tonic stimulus segment was $5 \mathrm{~min}$. The phasic conditioned stimulus was a circular red light that was produced by a multiple-stimulus projector, was $5 \mathrm{sec}$ in duration, and was located $50 \mathrm{~cm}$ in front of the subject's face. The electricshock unconditioned stimulus had an intensity of $4 \mathrm{~mA}$ and a duration of $1 \mathrm{msec}$ (Kimmel, King, Hudy, \& Gardner, 1980). The shock was administered via zinc electrodes to the volar surface of the subject's preferred arm.

\section{Procedure}

Data were collected in an IAC audiometric chamber, dimly illuminated by a 75-W shielded lamp. After the subject's nonpreferred hand was cleaned with acetone and the skin resistance and shock electrodes were attached, the subject was seated in a padded office chair and the instructions were read via intercom.

Two separate sessions, separated by 2 days, were administered. The order of the first three auditory tonic segments on the 1st day was as follows for all subjects: light alone, light and shock paired, and shock alone. Three additional tonic auditory segments, one with each type of phasic stimulus arrangement, were delivered on the 1 st day, in a different random order for each subject. Tone frequencies associated with the different types of tonic segments were also randomly determined for each subject. All auditory tonic segments during both sessions were separated by periods of $20 \mathrm{sec}$ of time-out from auditory stimuli and phasic events. Each tonic segment contained six phasic events (i.e., six lights alone, six shocks alone, or six paired lights and shock). The intertrial interval between phasic events varied between 35 and $65 \mathrm{sec}$, with a mean of $50 \mathrm{sec}$. Each tonic segment began with a 30 -sec period containing no phasic events.

In the second session, all subjects received the tonic segment containing paired light and shock first. The second and third tonic segments, consisting of either light or shock alone, were administered in a different random order for each subject. As during the first session, six phasic events were administered during the first three tonic segments in the second session. Three additional tonic auditory segments were run, one of each frequency, but with no phasic events.

\section{RESULTS}

The phasic SCR elicited by the light and the shock was defined as the peak change in log conductance within the time period beginning $1 \mathrm{sec}$ following the onset of the stimulus and ending 4 sec later (first-interval response). The mean SCR magnitude to the visual conditioned stimulus, averaged in two-trial blocks, is shown in the upper panel of Figure 1 segregated according to
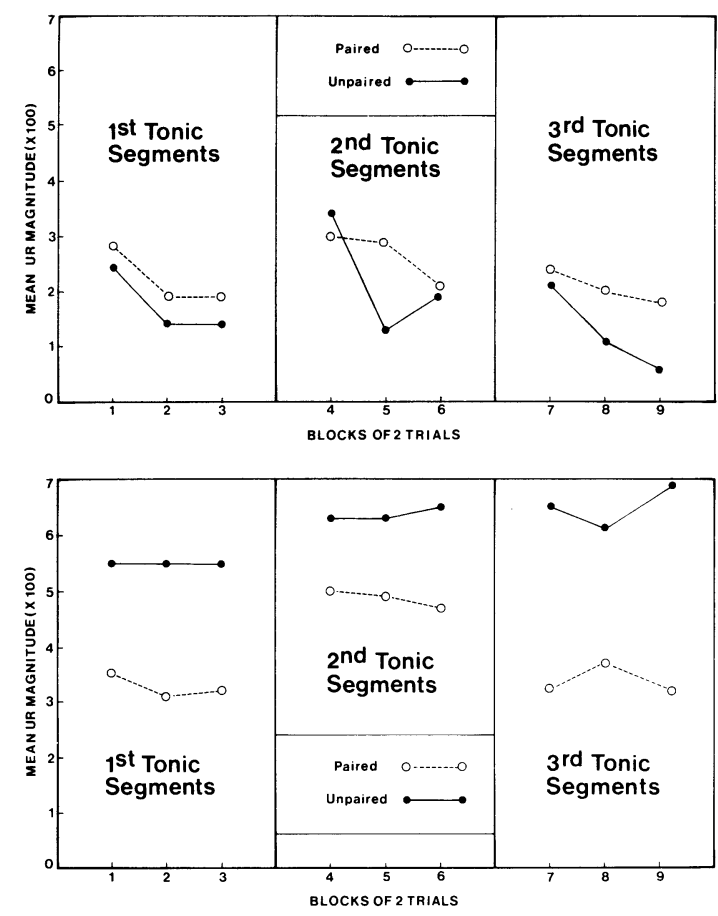

Figure 1. Average magnitude of conditioned response (upper panel) and unconditioned response (lower panel) in paired and unpaired tonic contexts, in blocks of two trials per tonic segment.

first, second, and third tonic auditory segments. The first two segments were administered in the first daily session, and the third segment was administered in the second daily session. The extinction trials (second session) are not included in Figure 1.

As is shown in Figure 1, the SCR to the visual stimulus tended to be larger in the tonic auditory context in which it was paired with shock than in the unpaired context, although SCR magnitude did not increase across training segments. Analysis of variance of these data indicated that the effect of pairing approached significance $[F(1,9)=4.86, E M S=.00030]$ and the within-segment decline in SCR magnitude was significant $[F(2,18)=7.97, E M S=.00024]$. No other effects were significant.

Because of the possibility that between-segment differentiation may have required repeated reconditioning and reextinction experiences within each segment, a second analysis of variance was done using only the second and third blocks of trials in each tonic segment (i.e., omitting the first two phasic trials in each segment) to estimate the pairing effect. In this analysis, the effect of pairing was highly significant $[F(1,9)=25.94$, EMS = .00008].

The mean SCR magnitude to the electric shock, averaged in two-trial blocks, is shown in the lower panel of Figure 1, segregated within the first, second, and third tonic auditory segments. As in the case of 
Table 1

Average Frequency of Unelicited Skin Conductance Responses per Minute During Initial Stimulus-Free Periods of Tonic Stimulus Presentation, During the Second Tonic Segments of Day 1 and the First Tonic Segments of Day 2

\begin{tabular}{cccc}
\hline & \multicolumn{3}{c}{ Tonic Stimulus } \\
\cline { 2 - 4 } Day & CS Only & CS + UCS & UCS Only \\
\hline 1 & 4.46 & 4.63 & 4.63 \\
2 & 3.07 & 4.90 & 5.66 \\
\hline
\end{tabular}

the conditioned responses, the unconditioned responses in the third tonic segments were obtained in the second daily session.

As Figure 1 clearly shows, the SCR to the shock was systematically smaller in the auditory context in which the shock was paired with light than in the unpaired context. This effect was shown to be significant by analysis of variance $[F(1,9)=8.80, E M S=.00270]$. When only the second and third blocks of trials in each segment were analyzed (omitting the first two shocks per segment), the pairing effect was even more significant $[(\mathrm{F} 1,9)=12.63, \mathrm{EMS}=.00131]$.

Since no phasic stimuli were administered during the first $30 \mathrm{sec}$ of each tonic stimulus, it was possible to examine the tonic frequency of unelicited SCRs during these periods (excluding the first $5 \mathrm{sec}$ so that phasic responses to the onset of the tonic stimulus would not be counted). In our previous research on transswitching (Kimmel \& Ray, 1978), we found that the frequency of unelicited SCRs differentiated significantly between tonic stimuli that had contained either paired or unpaired presentations of the conditioned and unconditioned stimuli.

Table 1 shows the average frequency of SCRs per minute during the last 25 of the first $30 \mathrm{sec}$ of each tonic stimulus, before any phasic stimuli were presented, during the second presentation of each tonic stimulus on the 1st day of conditioning and during their first presentation of the 2 nd day of conditioning. The earlier tonic segments are omitted because no tonic conditioned effects could be expected early in training; the later segments on the 2 nd day are omitted because these were extinction segments.

There was little difference among the three average SCR frequencies on the 1st day, as can be seen in Table 1. But, on the 2 nd day, there was a reduction in response frequency during the early part of the tonic stimulus, which contained only sporadic conditioned stimuli, and there was an increase in response frequency during the other two tonic stimuli, especially during the tonic stimulus that contained only unsignaled shocks. Analysis of variance of the unelicited SCR frequency data summarized in Table 1 indicated that the interaction suggested in the table did not attain significance $[F(2,18)=2.52, E M S=1.04]$. A separate analysis of only the 2 nd day also failed to yield a significant effect due to tonic stimuli $[F(2,18)=2.01$, EMS $=2.91]$.

\section{DISCUSSION}

The results of this study demonstrate the potential utility of the transswitching methodology as a means of studying withinsubjects conditioning effects. The administration of three different phasic stimulus arrangements within three different tonic stimulus contexts, as was done in this study, permits withinsubjects comparisons to be made of both conditioned and unconditioned responses under paired and unpaired conditions. Thus, the effect of pairing on conditioned response magnitude (i.e., conditioning) and on unconditioned response magnitude (i.e., diminution of the unconditioned response) were both shown. In addition, although differences in the frequency of unelicited responses that emerged in the different tonic contexts failed to attain statistical significance, it was of interest that a higher frequency of unelicited responding developed in the context in which shocks were administered alone, since the magnitude of the phasic response elicited by the unsignaled shock was shown to be larger than when the shock was signaled. We have also found (Kimmel, 1981) that tonic blood pressure changes in different tonic contexts are similar in direction to the phasic blood pressure responses elicited in those contexts. Thus, the present results tend to conform to the general proposition that tonic stimulus control of tonic levels or states may develop from differences in phasic reactions that have been elicited in these tonic contexts. The failure to achieve significant tonic differences in the present study is probably mainly a consequence of the small sample size employed.

\section{REFERENCES}

Asratyan, E. A. [Compensatory adaptation, reflex activity, and the brain] (S. A. Corson, trans.). New York: Oxford University Press, 1965.

KIMMEL, H. D. Inhibition of the unconditioned response in classical conditioning. Psychological Review, 1966, 73, 232-240.

Kimmel, H. D. The transswitching paradigm: A tool for studying the effect of context. In G. de Zeeuw \& P. van den Eeden (Eds.), Problems of context. Amsterdam: Boekhandel, 1979.

Kimmel, H. D. Phasic and tonic defensive conditioned reactions in human transswitching. In G. Adam, I. Meszaros, \& E. I. Banyai (Eds.), Advances in physiological science (Vol. 17). Brain and behavior. Budapest: Adademiai Kiado, 1981.

Kimmel, H. D., King, J., Hudy, J. J., \& Gardner, K. A. A mutual inductance shocker. Behavior Research Methods \& Instrumentation, 1980, 12, 605-606.

Kimmel, H. D., \& RAY, R. L. Transswitching: Conditioning with tonic and phasic stimuli. Journal of Experimental Psychology: General, 1978, 107 ,187-205.

(Received for publication October 1, 1981.) 